\title{
A Comparative Study of Gallic Acid, Ellagic Acid, Urolithin A, and Urolithin B with NF-кB Protein as Anti Type 2 Diabetes Mellitus by In Silico
}

\author{
Maulida Hikmaranti $^{1 *}$, Ajeng M. Astiyani ${ }^{1}$, Khairul M. Hasanah ${ }^{1}$, Nuril M. Maghfiroh ${ }^{1}$ \\ ${ }^{1}$ Biology Department, Faculty Mathematics and Natural Sciences, Brawijaya University \\ Jl Veteran, No 1, Malang, 65145, Indonesia
}

Submission: April 2020; Revised: May 2020; Accepted: May 2020

* Corresponding author: Maulida Hikmaranti; e-mail: maulidaranti@student.ub.ac.id; tel.: 081254698378

\begin{abstract}
Diabetes type 2 is a common disease with clinical symptoms of abnormal insulin secretion. One of the pathways involved in the pathogenic mechanism of Type 2 Diabetes Mellitus (T2DM) is NF- $\kappa B$ pathway. The walnuts contain natural compounds such as gallic acid, ellagic acid, urolithin $A$, urolithin $B$, and it is potential to be antioxidants and anti-inflammatory. In this research, we focus on the comparative study of 4 compounds as an inhibitor of NF- $\kappa B$ complex by molecular docking interactions between $N F-\kappa B$ and these ligands as anti-T2DM compounds. The method is preparation of $N F-\kappa B$ protein using Discovery Studio 4.1, preparation of ligand: gallic acid, ellagic acid, urolithin, and urolithin B using Pyrx software. After that, molecular docking of protein-ligand was conducted by using Hex 8.0.0 software, then visualized with Discovery Studio 2019 and then analyzed the bioactivity of the compound through Mollinspiration web, respectively. The result of Mollinspiration shows that 4 compounds have good quality as a drug based on the lipinski 5 rules. The docking results show that four compounds can actively bind to the active site of NF- $\kappa B$ with the different bond energies. Ellagic acid is the most stable compound and the highest activity to inhibit the NF- $\kappa B$ pathway because it has highest binding energy than the other $(-228,9 \mathrm{kcal} / \mathrm{mol})$. Ellagic acid is an active polyphenol compound that is good to use as an antidepressant. Based on these comparative studies, ellagic acid has the best potential among the three other compounds in inhibiting $N F-\kappa B$ activity. In addition, all compounds can effectively inhibit the activation and translocation of NF- $\mathrm{kB}$ from the cytoplasm to the nucleus so the $N F-\kappa B$ is unable to regulate DNA sequences that encode proinflammatory proteins and then be able to reduce the pathophysiological effects of type 2 diabetes mellitus.
\end{abstract}

Keywords: Ellagic acid, Gallic acid, T2DM, NF-кB, Urolithin A, Urolithin B.

\section{INTRODUCTION}

Diabetes mellitus is a metabolic disease that has a $2.1 \%$ prevalence rate in Indonesia. While developed countries in the world have 1,9\% prevalence. Diabetes mellitus is a disease that is stimulated by genetics, diet, ethnicity, and age which is characterized by blood in the blood that exceeds normal limits (> $200 \mathrm{mg} / \mathrm{dl}$ )[1]. There are 2 types of diabetes, namely diabetes 1 and type 2 diabetes (T2DM). Type 2 diabetes is more common than type 1 diabetes with a percentage of $90-95 \%$ more [2]. Type 2 diabetes has clinical symptoms in the form of abnormal insulin secretion. One potential pathogenic transition to T2DM is oxidative stress which is correlated with inflammation. One of the pathways involved in this relationship is the NF- $\kappa \mathrm{B}$ pathway [3].

The NF- $\kappa B$ activation pathway involves a protein cascade that regulates $\mathrm{NF}-\kappa \mathrm{B}$ translocation and activates it as a transcription factor. Blocking displacement paths to divert translocation from NF$\kappa \mathrm{B}$ can divert NF-KB activation. The NF- $\mathrm{BB}$ active complex consists of 2 subunits namely p65 and p50 subunits which have sequence homology at the $\mathrm{N}$ terminal position [4]. The protein inhibitor of NF- $\mathrm{kB}$ is IkB Kinase (IKK) which phosphorylates IkB. IkB will dissociate from NF- $\kappa B$ and cause NF- $\kappa B$ dimers to translocate to the nucleus and target genes which are pro-inflammatory cytokines that cause insulinrelated pathophysiology from various type 2 diabetes [5].

Walnuts (Juglans regia L.) is a type of nut that is rich in nutritional sources consisting of protein and essential fatty acids and high content of polyphenol compounds. The main polyphenol content in walnuts is ellagitannin which contains antioxidants that contain ellagic acid. Ellagic acid in the body can form into urothilin A and urothilin B by the large intestine which is also a combination of antioxidants. In addition to polyphenol content, gallic acid is also available which has potential effects as an antioxidant and anti-inflammatory. The content in walnuts can be used as a treatment for several diseases that are triggered by inflammation, one of which is type 2 diabetes mellitus [6]. 
Gallic acid has been shown to suppress inflammatory events by affecting the NF- $\kappa \mathrm{B}$ signaling pathway [7]. Meanwhile, Ellagic acid is also able to replace anti-inflammatory through oxidative reduction, which can overcome inflammation. When inflamed, there are several proteins involved, one of which is NF- $\mathrm{kB}$. The results of research through in vivo tests also prove that ellagic acid compounds can inhibit inflammation [8]. Therefore, this study aims to compare several compounds (gallic acid, ellagic acid, urothilin A, and urothilin B) as inhibitors of the NF- $\kappa B$ complex with molecular docking studies analysis between NF- $\kappa \mathrm{B}$ and these ligands as antiT2DM.

\section{METHODS}

\section{Proteins and Ligands Preparation}

The 3-dimensional structure of the protein, NF$\kappa B$ protein was taken from the RSCB PDB database with ID: 3GUT [4] and downloaded in PDB format. Removal of the water molecules, ligands, and visualization of proteins was conducted by Discovery Studio 4.1. The ligands used are gallic acid (GA) (CID: 370), ellagic acid (EA) (CID: 5281855), urolithin A (CID: 5488186), and urolithin B (CID: 5380406) in SDF format. The preparation of the compound was conducted by the reduction of its energy using Pyrx [9]. The active sites of NF- $\mathrm{BB}$ are carried out with literature studies.

\section{Docking and Analysis}

Docking interacts with NF- $\kappa$ SB receptors with gallic acid ligands, ellagic acid, urolithin A, urolithin B, using Hex 8.0.0 software and visualized with Discovery Studio 4.1 software. Bioactivity of compounds of each ligand is identified by accessing the information on the Mollinspiration web (http://www.mollinspiration.com) [9]. After that analysis was carried out based on the amount of binding energy and interactions between amino acid residues in the active sites of NF- $\mathrm{\kappa B}$ with ligands [4].

\section{RESULTS AND DISCUSSION}

Bioactivation of the four compounds namely gallic acid, ellagic acid, urolithin A, urolithin B can be seen from the results of the analysis on the Web Mollinsporation. The results show that these compounds have the potential to be used as drugs. Bioactivation of urolithin A compounds has the highest miLogPa value among other compounds, while gallic acid compounds have the smallest miLogPa value (Table 1). Based on the physicochemical properties of the four compounds obtained using Mollinspiration, the five compounds fulfill the criteria as drugs based on the 5 Lipinski rule. Thus, the five compounds are highly likely to be applied as drugs orally. All four compounds have a miLogPa value below 4 which indicates that the five compounds have a high permeability to cells. Urolithin A and Ellagic Acid have drug-like properties of nuclear receptors ligand and enzyme inhibitors [10].

Table 1. The physicochemical properties and biological activity prediction of ligands.

\begin{tabular}{cccc}
\hline Compond & miLogPa & $\begin{array}{c}\text { Nuclear } \\
\text { Receptor Ligand }\end{array}$ & $\begin{array}{c}\text { Inhibitor } \\
\text { Enzyme }\end{array}$ \\
\hline Urolithin A & 2.12 & 0.09 & 0.05 \\
Urolithin B & 2.62 & -0.03 & -0.03 \\
Ellagic Acid & 0.94 & 0.11 & 0.17 \\
Gallic Acid & 0.59 & -0.52 & -0.17 \\
\hline
\end{tabular}

According to Chemapalli (2019), using the CASTp program it was found that NF- $\kappa \mathrm{B}$ has active sites on the following amino acid residues : Arg33, Tyr36, Lys37, Cys38, Glu39, Arg124, Lya122, Asp125, Glu128, Ala129, Val121, Gln132, Asn186, Lys218, Lys218, Arg246, Tyr357, Val358, Glu360, His441, Val442, Lys123, Thr443, Lys444, Lys503, Glu504, Ser508, Lys541, Arg605 Gln606, Ala542. Gallic acid and $\mathrm{NF}-\kappa \mathrm{B}$ protein have binding energy $-169.1 \mathrm{kcal}$ /mol, respectively. Amino acid residues that bind between NF-kB-GA are Lys37, Cys120, Tyr36, Cys38, Lys122. NF-кB and EA showed that this compound was able to bind to the amino acid residue side of NF- $\kappa \mathrm{B}$ namely Lys $221, \mathrm{Gln} 220$, Arg24 with a bond energy of $-228.9 \mathrm{kcal} / \mathrm{mol}$. NF$\kappa \mathrm{B}$ and Urothilin A showed that this compound was capable of binding with several amino acid bond residues in NF- $\mathrm{BB}$ namely $\operatorname{Arg} 246$, Lys 221 with a bond energy of $-211.8 \mathrm{kcal} / \mathrm{mol}$. NF- $\mathrm{kB}$ and Urothilin B compound also showed that this compound was able to bind to several active residues of amino acid in NF- $\mathrm{kB}$ that are Arg246 and Lys 221 with a bond energy of $-204.8 \mathrm{kcal} / \mathrm{mol}$ (Table 2).

The active complex of NF- $\mathrm{BB}$ in hyperactive states capable of producing pro-inflammatory cytokines when it binds to DNA. The NF-kB signaling pathway involves the IKK kinase complex consisting of two kinases (IKK $\alpha$ and IKK $\beta$ ) and one regulatory subunit, NEMO / IKK $\gamma$. Through IKK $\beta$ phosphorylation, it activates the canonical signaling pathway which will phosphorylate IкB and marked for destruction by proteasome mediation in the cytosol. This causes the NF- $\kappa \mathrm{B}$ transcription 
factor to be activated. It is transcription factors that involve proinflammatory target genes, such as TNF- $\alpha$, IL-6, and IL- $\beta$ in the liver and adipose tissue. These anti-inflammatory cytokines will induce insulin resistance in the tissues where the proinflammatory cytokines are produced [3].
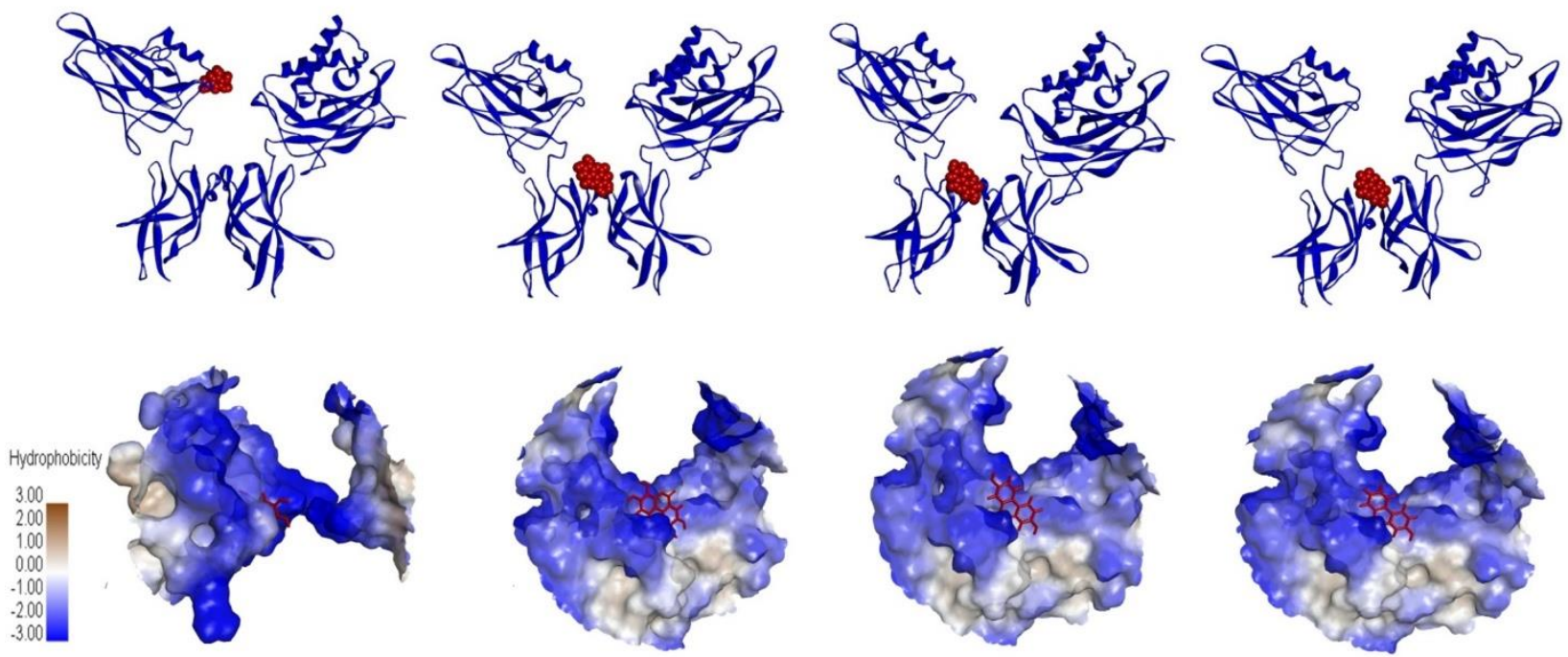

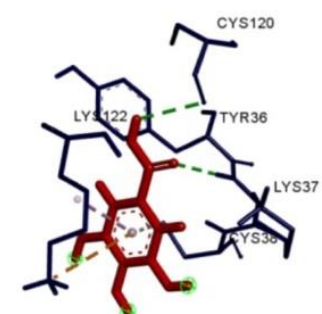

(a)

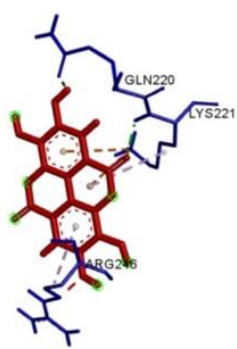

(b)

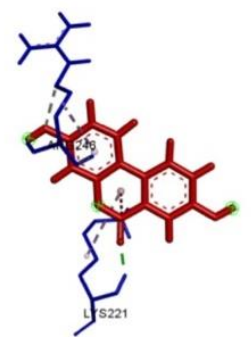

(c)

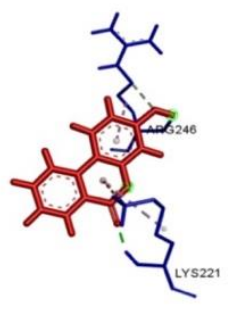

(d)

Figure 1. Interactions between NF- $\mathrm{KB}$ and ligands, a) Gallic Acid, b) Ellagic acid, c) Urothilin A, and d) Urothilin B. At the top, shows the interaction between NF-KB (blue band) with ligands (red ball). The middle section shows the hidrophobicity surface map of the NF-KB interactions with ligands and interactions between NF- $\mathrm{KB}$ and ligands are hydrophilic. At the bottom, shows the interaction of ligands with amino acid residues from NF-kB.

The NF- $\kappa \mathrm{B}$ complex consists of 2 subunits, namely p65 and p50 subunits which have a shared homology sequence at their N-terminal points. The I $\kappa \mathrm{B}$ binding site is in the $\mathrm{p} 65$ subunit which has a nuclear localization sequence (NLS). If the NLS site binds to I $\mathrm{B}$ B it will prevent the NF- $\kappa \mathrm{B}$ from translocating to the nucleus. Therefore, NLS can also be a target to inhibit NF- $\kappa \mathrm{B}$ translocation. Besides, a blockade of interactions between NF- $\mathrm{BB}$ and DNA in the nucleus can also be carried out to inhibit NF- $\mathrm{KB}$ activation (Figure 2) [4].

Gallic acid, ellagic acid, urolithin $\mathrm{A}$, and urolithin B. In this study can bind to $N F-\kappa B$ transcription factors. The compound that has the strongest tendency to bind to NF- $\kappa \mathrm{B}$ based on its binding, energy is ellagic acid. Then followed by urolithin A, urolithin B, and the last is gallic acid. The lower the binding energy value of the two molecules, the easier it is to bind. The lower of the value of free energy or delta $G$, it is considered close to the biological system [11]. In addition, this is also related to the number of aromatic rings possessed by compound, the better its ability to reduce inflammation in diabetes [12].

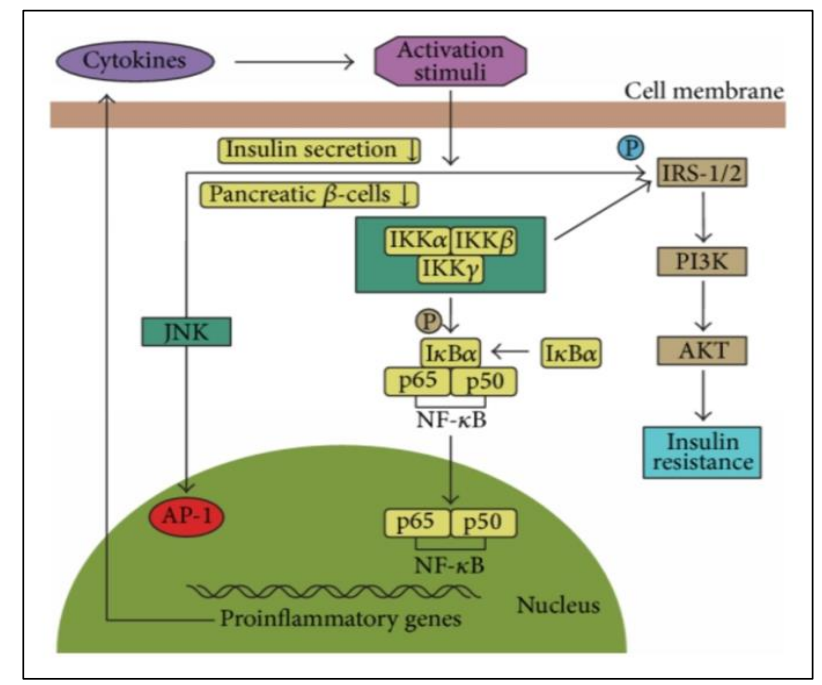

Figure 2. NF- $\kappa \mathrm{B}$ signaling pathway [5]. 
Table 2. Type of Bond Interaction, Binding Energy and Amino Acid Residues of NF- $\kappa$ B with Urolithin A, B, Ellagic Acid, and Gallic Acid.

\begin{tabular}{|c|c|c|c|c|c|}
\hline No & Protein-Ligand & Name & $\begin{array}{l}\text { Binding } \\
\text { Category }\end{array}$ & $\begin{array}{c}\text { Binding } \\
\text { Energy } \\
\text { (kcal/mol) }\end{array}$ & $\begin{array}{l}\text { Amino Acid Site } \\
\text { in NF-кB }\end{array}$ \\
\hline \multirow[t]{6}{*}{1.} & \multirow{6}{*}{$\begin{array}{l}\mathrm{NF}-\kappa \mathrm{B}-\text { Ellagic } \\
\text { acid }\end{array}$} & 1. A:LYS221:HN-:LIG1-O & 1. Hydrogen & \multirow[t]{6}{*}{ E:- 228.9} & \multirow[t]{6}{*}{ Lys221, Gln220, Arg246 } \\
\hline & & 2. :LIG1:H-A:GLN220:OE1 & 2. Hydrogen & & \\
\hline & & 3. A:LYS221:NZ-:LIG1 & 3. Electrostatic & & \\
\hline & & 4. A:LYS221:NZ-:LIG1 & 4. Electrostatic & & \\
\hline & & 5. :LIG1-A:LYS221 & 5. Hydrophobic & & \\
\hline & & 6. :LIG1-A:ARG246 & 6. Hydrophobic & & \\
\hline \multirow[t]{5}{*}{2.} & $\mathrm{NF}-\kappa \mathrm{B}-$ & 1. A:LYS221:HN-:LIG1:O & 1. Hydrogen & \multirow[t]{5}{*}{$\mathrm{E}:-211.8$} & \multirow[t]{5}{*}{ Arg246, Lys 221} \\
\hline & \multirow[t]{4}{*}{ Urolithin A } & 2. A:ARG246:CD-:LIG1:O & 2. Hydrogen & & \\
\hline & & 3. A: LYS221:NZ-LIG1 & 3. Electrostatic & & \\
\hline & & 4. :LIG1-A:LYS221 & 4. Hydrophobic & & \\
\hline & & 5. :LIG1-A:ARG246 & 5. Hydrophobic & & \\
\hline \multirow[t]{5}{*}{3.} & $\mathrm{NF}-\kappa \mathrm{B}-$ & 1. A:LYS221:HN-:LIG1:O & 1. Hydrogen & \multirow[t]{5}{*}{$\mathrm{E}:-204.8$} & \multirow[t]{5}{*}{ Arg246, Lys 221} \\
\hline & \multirow[t]{4}{*}{ Urolithin B } & 2. A:ARG246:CD-:LIG1:O & 2. Hydrogen & & \\
\hline & & 3. A: LYS221:NZ-LIG1 & 3. Electrostatic & & \\
\hline & & 4. :LIG1-A:LYS221 & 4. Hydrophobic & & \\
\hline & & 5. :LIG1-A:ARG246 & 5. Hydrophobic & & \\
\hline \multirow[t]{5}{*}{4.} & $\mathrm{NF}-\kappa \mathrm{B}-$ Gallic & 1. A:LYS37:HN-:LIG1:O & 1. Hydrogen & \multirow[t]{5}{*}{$\mathrm{E}:-169.1$} & \multirow{5}{*}{$\begin{array}{l}\text { Lys37,Cys120,Tyr36, } \\
\text { Cys38, Lys122 }\end{array}$} \\
\hline & \multirow[t]{4}{*}{ Acid } & 2. LIG1:H-A:CYS120:O & 2. Hydrogen & & \\
\hline & & 3. A: LYS122:NZ-LIG1 & 3. Electrostatic & & \\
\hline & & 4. A:CYS38:SG-:LIG1 & 4. Hydrogen & & \\
\hline & & 5. :LIG1-A:LYS122 & 5. Hydrophobic & & \\
\hline
\end{tabular}

Gallic acid, ellagic acid, urolithin A, and urolithin B bind to the active site of NF- $\mathrm{kB}$. This indicates that the four ligands potentially can inhibit $\mathrm{NF}-\mathrm{\kappa B}$ protein activity. Ellagic acid, urolithin A, and urolithin B interact as hydrophobic bonds with the amino acid residue of Arg246. Meanwhile, ellagic acid binds to the active sites of NF- $\mathrm{kB}$ (Tyr36, Lys37, Cys38, Lys122) with hydrogen, hydrophobic, and electrostatic bonds (Table 2).

Insulin resistance can occur due to translocation of NF- $\kappa \mathrm{B}$ protein from the cytoplasm to the nucleus which causes the activation of several proinflammatory cytokines. Therefore, inhibition of NF- $\kappa \mathrm{B}$ active sites is needed to prevent the translocation. The compounds tested were gallic acid, ellagic acid, urolithin A and urolithin B showed that the four could act as anti-inflammatory compounds by inhibiting the activity of NF- $\kappa \mathrm{B}$ complex with DNA. The interactions analysis between ligands and NF- $\kappa B$ protein does not involve active sites in NLS sites based on the known active sites of NLS [4]. If the binding involves several active sites of amino acid residues called NLS which will bind to one of the subunits of NF- $\mathrm{BB}$, which can prevent translocation of NF- $\kappa B$ to the nucleus so that [13].
The four docking compounds proved that these compounds could potentially bind DNA and prevent $\mathrm{NF}-\mathrm{kB}$ to regulate the gene that encodes proinflammatory cytokines. Thus, those ligands potentially initiate anti-inflammatory mechanisms. These interactions can work by increasing the potential of compounds in the specific inhibition of the active sites of the amino acid residues [13]. Several previous studies, carried out in vivo also stated that urolithin A and urolithin B were able to act as anti-inflammatory compounds simultaneously by inhibiting the activation of NF- $\kappa \mathrm{B}$ by preventing the unification of subunits at one of the consensus binding sites. It is also supported because of its relation to the process mediated by microbiota in the intestine. However, the mechanism of interaction in the large intestine has not been examined further [14].

Ellagic acid (C14H6O8) is a natural phenolic compound present in some fruits and vegetables. High levels of EA found in walnut. EA has been reported to have some biological activities, including antioxidant, anti-carcinogenic, antifibrosis, and antiproliferative properties as observed in some of the in vitro (cell culture) and in vivo (OA mouse model) [15]. Ellagic acids have a chemoprotective 
effect in cellular models by inhibiting reactive chemical carcinogens [e.g., nitrosamines and polycyclic aromatic hydrocarbons] from covalently modifying DNA and free radical scavenging activities [16]. Also, EA exhibits plenty of bioactive effects including cardio-protection, anti-diabetic, anti-proliferative effects in cancer, antioxidative stress, anti-inflammatory, and performing a neuroprotective role [17]. Ellagic acid can decrease and inhibits IL-1 $\beta$-induced inflammation by suppressing the activation NF- $\kappa \mathrm{B}$ pathway.

\section{CONCLUSION}

Ellagic acid has the best ability to inhibit NF- $\mathrm{BB}$ activity. The ellagic acid compound has the highest binding energy, which showed that ellagic acid compounds are the most stable compounds and have the highest activation to inhibition $\mathrm{NF}-\kappa \mathrm{B}$ pathway. From that, it can be concluded that ellagic acid has the most effectively controlling the activation of NF-kB from the cytoplasm to the nucleus through the binding of ligands to amino acid residues from the active site NF- $\mathrm{kB}$ complex. It plays a role to prevent $\mathrm{NF}-\kappa \mathrm{B}$ translocation from the cytoplasm to the nucleus. Therefore, NF- $\kappa \mathrm{B}$ is not able to regulate DNA sequences that encode proinflammatory proteins so it can reduce the pathophysiological effects of type 2 diabetes mellitus.

\section{ACKNOWLEDGEMENTS}

The authors are sending our thanks to Prof. Fatchiyah, Ph.D., Prof. Widodo, S.Si., M.Si., Ph.D.Med.Sc., Nia Kurniawan, S.Si, M.P., D.Sc., and Yoga Dwi Jatmiko, S.Si., M.App.Sc., Ph.D. and the laboratory assistants for their guidance on our project.

\section{REFERENCES}

1. Arifin, B., Idrus, L. R., Van Asselt, A. D., Purba, F. D., Perwitasari, D. A., Thobari, J. A., Postma, M. J. 2019. Health-related quality of life in Indonesian type 2 diabetes mellitus outpatients measured with the Bahasa version of EQ-5D. Quality of Life Research. 28(5): 1179-1190.

2. Tsalamandris, S., Antonopoulos, A. S., Oikonomou, E., Papamikroulis, G. A., Vogiatzi, G., Papaioannou, S. 2019. The role of inflammation in diabetes: current concepts and future perspectives. European Cardiology Review. 14(1): 1-50.

3. Donath, M. Y., Shoelson, S. E. 2011. Type 2 diabetes as an inflammatory disease. Nature Reviews Immunology. 11(2): 98-107.
4. Cheemanapalli, S., Chinthakunta, N., Shaikh, N. M., Shivaranjani, V., Pamuru, R.R., Chitta, S.K. 2019. Comparative binding studies of curcumin and tangeretin on up-stream elements of NF-kB cascade: a combined molecular docking approach. Network Modeling Analysis in Health Informatics and Bioinformatics. 8(1): 1-15.

5. Chen, L., Chen, R., Wang, H., Liang, F. 2015. Mechanisms linking inflammation to insulin resistance. International journal of endocrinology. 2015:1-9

6. Sánchez-González C, Ciudad CJ, Noé V, Izquierdo-Pulido M. 2017. Health benefits of walnut polyphenols: An exploration beyond their lipid profile. Crit Rev Food Sci Nutr. 57(16):3373-3383.

7. Zhu, L., Gu, P., Shen, H. 2019. Gallic acid improved inflammation via NF- $\mathrm{B}$ p pathway in TNBS-induced ulcerative colitis. International immunopharmacology. 67:129-131

8. Roy, S., D. Bagchi., S. P. Raychaudhuri. 2012. Chronic Inflammation: Molecular Pathophysiology, Nutritional and Therapeutic Interventions. CRC Press, New York

9. Sari, DRT., Ketudat-Cairns, JR., Safitri, A., Fatchiyah, F. 2019. Virtual Prediction of the Delphinidin-3-O-glucoside and Peonidin-3-Oglucoside as Anti-inflammatory of TNF- $\alpha$ Signaling. Acta Informatica Medica. 27(3): 152157.

10. Alodeani, E.A., Arshad, M, Izhari, M.A. 2015. Anti-uropathogenic activity, drug likeness, physicochemical and molecular docking assessment of (E-)-N'-(substitutedbenzylidene)-2-(quinolin-8-ylox) acetohydrazide. Asian Pacific Journal of Tropical Biomedicine. 5(8):676-683.

11. Elokely, K.M., Doerksen, R.J. 2013. Docking Challenge: Protein Sampling and Molecular Docking Performance. Journal of Chemical Information and Modeling. 53(8): 1934-1945

12. Adefegha, SA., Oyeleye, SI., Oboh, G. 2015. Distribution of Phenolic Contents, Antidiabetic Potentials, Antihypertensive Properties, and Antioxidative Effects of Soursop (Annona Muricata L.) Fruit Parts In Vitro. Biochem. Research international. 2015:1-8.

13. Giridharan, S., Srinivasan, M. 2018. Mechanisms of NF- $\mathrm{kB}$ p65 and strategies for therapeutic manipulation. Journal of inflammation research. 11:407-419

14. Sarrias., AG., Larosa., M., Barberin, FA., Dolara, P. 2010. NF-Kb dependent AntiInflammatory Activity of Urolithin, Gut Microbiota Ellagic Acid-Derived Metabolites, In Human Colonic Fibrobalst. British Journal of Nutrition. 104:503-512. 